\title{
Breathers in Josephson junction ladders: Resonances and electromagnetic wave spectroscopy
}

Miroshnichenko, A. E.; Flach, S.; Fistul, M.; Zolotaryuk, Yaroslav; Page, J. B.

Published in:

Physical Review E. Statistical, Nonlinear, and Soft Matter Physics

Link to article, DOI:

10.1103/PhysRevE.64.066601

Publication date:

2001

Document Version

Publisher's PDF, also known as Version of record

Link back to DTU Orbit

Citation (APA):

Miroshnichenko, A. E., Flach, S., Fistul, M., Zolotaryuk, Y., \& Page, J. B. (2001). Breathers in Josephson junction ladders: Resonances and electromagnetic wave spectroscopy. Physical Review E. Statistical, Nonlinear, and Soft Matter Physics, 64(6), 066601. https://doi.org/10.1103/PhysRevE.64.066601

\section{General rights}

Copyright and moral rights for the publications made accessible in the public portal are retained by the authors and/or other copyright owners and it is a condition of accessing publications that users recognise and abide by the legal requirements associated with these rights.

- Users may download and print one copy of any publication from the public portal for the purpose of private study or research.

- You may not further distribute the material or use it for any profit-making activity or commercial gain

- You may freely distribute the URL identifying the publication in the public portal 


\title{
Breathers in Josephson junction ladders: Resonances and electromagnetic wave spectroscopy
}

\author{
A. E. Miroshnichenko, S. Flach, and M. V. Fistul \\ Max-Planck-Institut für Physik komplexer Systeme, Nöthnitzer Strasse 38, D-01187 Dresden, Germany \\ Y. Zolotaryuk \\ Group of Mathematical Physics, IMM, Technical University of Denmark, Building 321, Richard Petersens Plads, DK-2800 Kgs. Lyngby, \\ Denmark \\ J. B. Page \\ Department of Physics and Astronomy, Arizona State University, Tempe, Arizona 85287-1504
}

(Received 13 March 2001; published 7 November 2001)

\begin{abstract}
We present a theoretical study of the resonant interaction between dynamical localized states (discrete breathers) and linear electromagnetic excitations (EE's) in Josephson junction ladders. By making use of direct numerical simulations we find that such an interaction manifests itself by resonant steps and various sharp switchings (voltage jumps) in the current-voltage characteristics. Moreover, the power of ac oscillations away from the breather center (the breather tail) displays singularities as the externally applied dc bias decreases. All these features may be mapped to the spectrum of EE's that has been derived analytically and numerically. Using an improved analysis of the breather tail, a spectroscopy of the EE's is developed. The nature of breather instability driven by localized EE's is established.
\end{abstract}

DOI: 10.1103/PhysRevE.64.066601

PACS number(s): 63.20.Ls, 05.45.Yv, 74.50.+r

\section{INTRODUCTION}

Various nonlinear and discrete systems (nonlinear lattices) have attracted a lot of interest as they display diverse fascinating phenomena [1]. Well-known examples of such phenomena are solitary excitations, propagation of (non)linear waves, and the appearance of various inhomogeneous structures.

Moreover, the interest in this area was boosted by the prediction, theoretical analysis [2-4], and the subsequent observation [5-10] of intrinsic dynamic localized excitations (discrete breathers) that are periodic in time and localized in space. Note here that the origin of such dynamical localization is not the presence of disorder but the interplay between the nonlinearity and discreteness.

These peculiar states have been experimentally verified as vibrational modes in low-dimensional crystals [5], localized excitations in spin lattices [6], and localized resistive states in Josephson junction arrays [7-10]. The latter systems are of special interest because they have served for many years as well-controlled laboratory objects to study various nonlinear phenomena $[1,11]$. Moreover, at variance with other systems, intrinsic localized modes found in Josephson coupled systems may be excited in the presence of time-independent external driving forces.

A well-known structure where dynamical localized states appear, is the anisotropic Josephson junction ladder (JJL) [7-10]. A schematic view of such a ladder is given in Fig. 1. The ladder contains small Josephson junctions indicated by crosses in Fig. 1, in both longitudinal (vertical junctions) and transverse (horizontal junctions) directions to the dc bias current $\gamma$. The anisotropy of the ladder is due to the different sizes of vertical and horizontal junctions and is characterized by the anisotropy parameter $\eta=I_{c H} / I_{c V}$, where $I_{c V}$ and $I_{c H}$ are, respectively, the critical currents of the vertical and horizontal junctions.

Dynamical localized excitations persist in a JJL due to the intrinsic bistability property of a single small underdamped Josephson junction. One of these stable states is a superconducting one with zero voltage drop across the junction. The other state is a resistive one with a nonzero voltage drop, also called a whirling state. A breather state in a JJL is characterized by a few junctions being in the resistive state, while the rest of all junctions are in the superconducting state. The presence of breather states may be verified by measurements of a total de voltage drop across the ladder, which is used to plot current-voltage $(I-V)$ characteristics. This method does not provide spatially resolved information. It has been successfully combined with snapshots made using lowtemperature laser microscopy techniques [8], which allow for a spatial resolution of the dc voltage drops. Note that both methods provide only time-averaged voltage drop data, so the internal dynamics are so far not accessible in experiments.

However, the full dynamical picture is much more subtle than the time-averaged picture might suggest. In particular, the Josephson junctions in the superconducting state exhibit small librations of the Josephson phase and correspondingly, nonzero ac voltage drops. The amplitude of these librations should decay to zero with increasing distance from the resis-

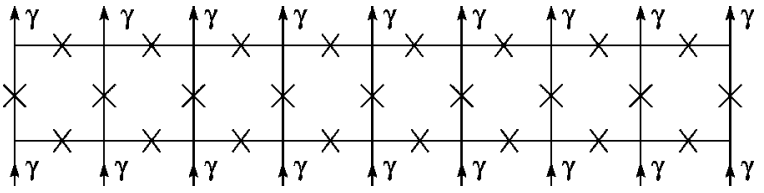

FIG. 1. Josephson junction ladder. Crosses mark the individual junctions. Arrows indicate the direction of external current flow (dc bias $\gamma)$. 
tive junctions. It is also well known that various systems of coupled Josephson junctions support a delocalized class of excitations, namely, small amplitude electromagnetic waves (EW's) $[11,12]$. In JJL's, the spectrum of EW's consists of three branches and depends in a complex manner on the anisotropy $\eta$ and the dc bias $\gamma$. The resonant interaction of these EW's with the homogeneous whirling state (HWS) in the presence of an externally applied magnetic field has been studied in Ref. [12]. It was shown that such an interaction leads to resonant steps in $I-V$ curves and the voltage positions of resonant steps may be mapped onto the spectrum of EW's.

Early theoretical studies [13] dealt with the possibility of resonant interaction of breather states with EW's of the ladder. Due to the intrinsic spatial inhomogeneity of a breather state, EW's may be excited even in the absence of an externally applied magnetic field. The resonant interaction of EW's with the breather state manifests itself through the appearance of resonant steps [10] and various switchings between different breather states ("voltage jumps") in $I-V$ curves. Moreover, the amplitude of the Josephson phase librations at some distance from the breather center increases drastically due to this interaction [13].

The spatial inhomogeneity of a breather state allows also for the appearance of localized small amplitude electromagnetic excitations (EE's). We will show that the presence of localized EE's and their resonant interaction with the breather is of crucial importance. Our study resolves a longstanding puzzle of the nature of breather instabilities. We show that most of these instabilities are driven by localized EE's. Especially the so-called retrapping (i.e., the switching from a breather to the superconducting state) is due to these instabilities and cannot be explained using standard retrapping arguments.

In this paper, we present a consistent theoretical study of resonant interactions between the breather states and EE's. We will derive the spectrum of EW's and calculate the ac power of oscillations at some distance from the breather center ("breather tail"). By making use of direct numerical simulations of the dynamics of JJL's in different parameter ranges, we demonstrate that the $I-V$ curves display a variety of different resonance steps and voltage jumps as the external dc bias, and correspondingly, the breather frequency, decrease. All these features are mapped onto various resonances of the breather frequency (or even its second and third harmonics) with EE frequencies, as well as with combinations of frequencies of different EE's. Moreover, by monitoring the power of ac oscillations as a function of the dc bias $\gamma$, we develop a spectroscopy of the EW's in the JJL.

The paper is organized as follows: In Sec. II, we derive the equations of motion within the framework of the resistively shunted junction (RSJ) model [11] and obtain the spectrum of linear EW's of a JJL. A symmetry classification of different types of breathers is presented in Sec. III. An improved analysis of the breather tail and the corresponding dependence of the power of ac oscillations on the breather frequency is given in Sec. IV. In Sec. V, we will classify different resonances of breathers with EE's. Direct numerical simulations of the dynamics of breather states are presented and discussed in Sec. VI. Section VII is devoted to the interpretation of the obtained resonances and switchings.

\section{DYNAMICS OF A JJL AND THE SPECTRUM OF LINEAR ELECTROMAGNETIC WAVES}

The complete dynamics of a JJL is determined by the time-dependent Josephson phases of vertical $\phi_{n}^{v}$, upper horizontal $\phi_{n}^{h}$, and lower horizontal $\widetilde{\phi}_{n}^{h}$ junctions. The subscript $n$ labels the cell number. By making use of the RSJ model for each junction [11] we obtain the following set of equations:

$$
\begin{gathered}
\mathcal{N}\left(\phi_{n}^{v}\right)=I_{n}^{v}, \\
\mathcal{N}\left(\phi_{n}^{h}\right)=\frac{1}{\eta} I_{n}^{h}, \\
\mathcal{N}\left(\widetilde{\phi}_{n}^{h}\right)=\frac{1}{\eta} \widetilde{I}_{n}^{h},
\end{gathered}
$$

where the nonlinear operator $\mathcal{N}$ is defined as

$$
\mathcal{N}(y)=\ddot{y}+\alpha \dot{y}+\sin y .
$$

Here, the unit of time is the inverse plasma frequency $\omega_{p}^{-1}$, and the currents $I_{n}^{v}, I_{n}^{h}$, and $\widetilde{I}_{n}^{h}$ are measured in units of the critical current of vertical junctions. The dimensionless parameter $\alpha$ determines the damping strength in each junction. Note that the positive current direction is chosen to be directed from bottom to top and from left to right. The currents flowing via the Josephson junctions and the Josephson phases are governed by the Kirchhoff laws

$$
\begin{aligned}
& \gamma=I_{n}^{v}+I_{n}^{h}-I_{n-1}^{h}, \\
& \gamma=I_{n}^{v}-\widetilde{I}_{n}^{h}+\widetilde{I}_{n-1}^{h},
\end{aligned}
$$

and the flux quantization law in each cell,

$$
-\beta_{L} I_{n}^{m}=\phi_{n}^{h}+\phi_{n+1}^{v}-\widetilde{\phi}_{n}^{h}-\phi_{n}^{v} .
$$

Here, we introduced the mesh currents $I_{n}^{m}$ and the normalized inductance of the cell, $\beta_{L}$.

The Kirchhoff equations may be subtracted from each other yielding

$$
I_{n}^{h}+\widetilde{I}_{n}^{h}=C,
$$

where $C$ is a constant for the whole ladder. This constant corresponds to the net difference between the currents flowing through the upper- and lower-horizontal junctions. For an open ladder of finite size, $C$ is zero. For a ladder of annular geometry with periodic boundary conditions $C$ may be nonzero and corresponds to the flux "trapped" by the ladder ring. In the following, we will consider the case of a finite open ladder with $C=0$. Then we may always eliminate the currents $\widetilde{I}_{n}^{h}$ from the set of equations (1) as $\widetilde{I}_{n}^{h}=-I_{n}^{h}$. Since 
the junction width is larger than the London penetration depth, the mesh currents are [14]

$$
I_{n}^{m}=I_{n}^{h},
$$

and the currents flowing through the vertical junctions are expressed as

$$
I_{n}^{v}=\gamma-I_{n}^{m}+I_{n-1}^{m} .
$$

Inserting the relations (4), (6), and (7) into Eq. (1) we finally obtain the following set of coupled differential equations $[8,12,13,15]$ :

$$
\begin{gathered}
\ddot{\phi}_{n}^{v}+\alpha \dot{\phi}_{n}^{v}+\sin \phi_{n}^{v}=\gamma+\frac{1}{\beta_{L}}\left(\Delta \phi_{n}^{v}+\nabla \phi_{n-1}^{h}-\nabla \widetilde{\phi}_{n-1}^{h}\right), \\
\ddot{\phi}_{n}^{h}+\alpha \dot{\phi}_{n}^{h}+\sin \phi_{n}^{h}=-\frac{1}{\eta \beta_{L}}\left(\nabla \phi_{n}^{v}+\phi_{n}^{h}-\widetilde{\phi}_{n}^{h}\right), \\
\ddot{\widetilde{\phi}}_{n}^{h}+\alpha \dot{\widetilde{\phi}}_{n}^{h}+\sin \widetilde{\phi}_{n}^{h}=\frac{1}{\eta \beta_{L}}\left(\nabla \phi_{n}^{v}+\phi_{n}^{h}-\widetilde{\phi}_{n}^{h}\right),
\end{gathered}
$$

where we use the notations $\Delta f_{n} \equiv f_{n-1}-2 f_{n}+f_{n+1}$ and $\nabla f_{n} \equiv f_{n+1}-f_{n}$.

Next, we carry out the analysis of the delocalized class of excitations, namely, of small amplitude electromagnetic waves (EW's). Note here that the spectrum of EW's $\omega(q)$ depends crucially on the state of the system. In the following, we will consider a static (superconducting) state, $\phi_{n}^{* v}$ $=\arcsin \gamma$ and $\phi_{n}^{* h}=\widetilde{\phi}_{n}^{* h}=0$. We decompose the Josephson phases into the particular form

$$
\begin{aligned}
& \phi_{n}^{v}=\phi_{n}^{* v}+\varphi_{n}^{v}, \\
& \phi_{n}^{h}=\phi_{n}^{* h}+\varphi_{n}^{h}, \\
& \widetilde{\phi}_{n}^{h}=\widetilde{\phi}_{n}^{* h}+\widetilde{\varphi}_{n}^{h},
\end{aligned}
$$

where $\varphi_{n}^{v}, \varphi_{n}^{h}$, and $\widetilde{\varphi}_{n}^{h}$ describe the small amplitude EW's. Substituting these expressions into system (8) and using the smallness of the amplitude of EW's we obtain

$$
\begin{gathered}
\ddot{\varphi}_{n}^{v}+\alpha \dot{\varphi}_{n}^{v}+\sqrt{1-\gamma^{2}} \varphi_{n}^{v}=\frac{1}{\beta_{L}}\left(\Delta \varphi_{n}^{v}+\nabla \varphi_{n-1}^{h}-\nabla \tilde{\varphi}_{n-1}^{h}\right), \\
\ddot{\varphi}_{n}^{h}+\alpha \dot{\varphi}_{n}^{h}+\varphi_{n}^{h}=-\frac{1}{\eta \beta_{L}}\left(\nabla \varphi_{n}^{v}+\varphi_{n}^{h}-\tilde{\varphi}_{n}^{h}\right), \\
\ddot{\widetilde{\varphi}}_{n}^{h}+\alpha \dot{\tilde{\varphi}}_{n}^{h}+\tilde{\varphi}_{n}^{h}=\frac{1}{\eta \beta_{L}}\left(\nabla \varphi_{n}^{v}+\varphi_{n}^{h}-\tilde{\varphi}_{n}^{h}\right) .
\end{gathered}
$$

In the weakly damped case as the parameter $\alpha \ll 1$, we can derive the spectrum $\omega(q)$ of EW's neglecting effects of dissipation. By taking the Josephson phases $\varphi_{n}^{v}, \varphi_{n}^{h}$, and $\widetilde{\varphi}_{n}^{h}$ in the form

$$
\left(\begin{array}{c}
\varphi_{n}^{v} \\
\varphi_{n}^{h} \\
\tilde{\varphi}_{n}^{h}
\end{array}\right)=e^{i(q n+\omega t)}\left(\begin{array}{c}
\Delta_{v} \\
\Delta_{h} \\
\widetilde{\Delta}_{h}
\end{array}\right)
$$

we find that the spectrum consists of three branches. The first is given by

$$
\omega_{0}^{2}=1, \quad \Delta_{v}=0, \quad \Delta_{h}=\tilde{\Delta}_{h} .
$$

This branch is dispersionless and EW's corresponding to this branch are characterized by nonactive vertical junctions and in phase (symmetric) librations of the Josephson phases of upper- and lower-horizontal junctions.

The two other solutions are generalizations of those discussed in Ref. [13], namely,

$$
\begin{gathered}
\omega_{ \pm}^{2}=F \pm \sqrt{F^{2}-G}, \\
F=\frac{1}{2}+\frac{1}{\beta_{L} \eta}+\frac{1}{2} \sqrt{1-\gamma^{2}}+\frac{1}{\beta_{L}}(1-\cos q), \\
G=\left(1+\frac{2}{\beta_{L} \eta}\right) \sqrt{1-\gamma^{2}}+\frac{2}{\beta_{L}}(1-\cos q) .
\end{gathered}
$$

Both branches have a nonzero dispersion.

The branch $\omega_{+}$is characterized by $\Delta_{h}=-\widetilde{\Delta}_{h}$ for all wave-vectors $q$, i.e., the upper- and lower-horizontal phases are antisymmetric. The frequency range of the branch is above the degenerate branch $\omega_{0}$, i.e., $\omega_{+}(q)>\omega_{0}$ and it depends strongly on $\beta_{L}$. As the parameter $\beta_{L}$ increases, the width of $\omega_{+}(q)$ decreases and the branch approaches the dispersionless one, $\omega_{0}$. In the opposite case of small $\beta_{L}$, the frequencies $\omega_{+}(q)$ increase as $1 / \sqrt{\beta_{L}}$. For zero wavenumber $q=0$, the amplitudes of EW's in this branch are characterized by $\Delta_{v}=0$ and $\Delta_{h}=-\widetilde{\Delta}_{h}$, which means that only horizontal junctions are excited.

The branch $\omega_{-}$becomes dispersionless for the particular case of $\gamma=0$ and it corresponds to the dispersionless band obtained in Ref. [13]. The frequency range of this branch is located below $\omega_{0}$, i.e., $\omega_{-}(q)<\omega_{0}(q)$. For zero wavenumber $q=0$, the horizontal junctions are not active $\left(\Delta_{h}\right.$ $=\widetilde{\Delta}_{h}=0$ ) and only vertical junctions are excited.

For a finite-size ladder with open boundary conditions and $N$ cells, i.e., $N+1$ vertical junctions, the spectrum of linear waves is discrete and characterized by the following choice of allowed wave number values:

$$
q_{l}=\frac{l \pi}{N+1}, \quad l=0,1,2, \ldots, N .
$$

These EW's are cavity modes of the JJL. Odd values of $l$ correspond to antisymmetric eigenvectors (with respect to reflections at the center of the ladder), whereas even values correspond to symmetric ones.

Note that the above spectrum of EW's (13) is in general quite different from the EW spectrum of the homogeneous whirling state [12]. The latter may be obtained by choosing 


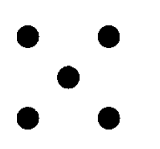

a)

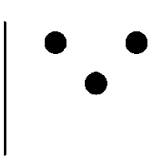

b)

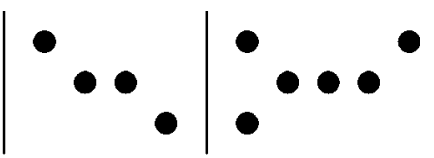

c) d)
FIG. 2. Examples of different types of breathers: (a) up-down symmetry, (b) left-right symmetry, (c) inversion symmetry, (d) no symmetry. Black spots indicate the positions of whirling junctions.

$\gamma=1$ in Eq. (13). The main difference is the appearance of a gapless (acoustic) lower branch.

\section{SYMMETRIES AND DC BIAS DEPENDENT FREQUENCIES OF BREATHERS}

In this section, we turn to the analysis of dynamic localized excitations (breathers) in JJL's. As mentioned in the introduction, breather states correspond to a few junctions being in the resistive state with all other junctions being in the superconducting state. The Josephson phases of resistive junctions are unbounded in time and the Josephson phases of superconducting junctions display small amplitude librations with a frequency $\Omega$. This frequency is called the breather frequency.

Experiments [7-10] have revealed many different breather structures. All of them can be classified into three symmetry types using the reflection symmetries of the JJL. Some possible realizations are presented schematically in Fig. 2.

Breathers from the first group reveal an "up-down' reflection symmetry $\hat{S}_{u d}$ [see, Fig. $\left.2(\mathrm{a})\right]$, i.e., they are invariant under exchange of upper- and lower-horizontal junctions. The second group consists of breathers invariant under a "'left-right', reflection symmetry $\hat{S}_{l r}$ [see, Fig. 2(b)], i.e., they are invariant under a reflection at a vertical line cutting the ladder (this line is located either in the middle between two vertical junctions, or passes directly through one vertical junction). A third distinct group of breathers possesses an “inversion" symmetry $\hat{S}_{\text {in }}$ (Fig. 2(c)), i.e., these breathers are invariant under a reflection at a point that is either located on a vertical junction or in the center of a plaquette. A fourth group of breathers has no symmetries at all and does not belong to any of the three listed symmetry types. A particular example of a breather without symmetry is shown in Fig. 2(d). All of these types of breather excitations have been observed experimentally and numerically [7-10]. Each group of breathers may also have a different number of vertical junctions in the resistive state. Note that the particular example in Fig. 2(a) possesses not only $\hat{S}_{u d}$ symmetry, but also $\hat{S}_{l r}$ and $\hat{S}_{i n}$ symmetries. However, it is also possible to construct more complex breather states that display $\hat{S}_{u d}$ symmetry only.

Next, we derive the average voltage drop across a resistive vertical junction $V=\left\langle\dot{\phi}^{v}\right\rangle$ for different breather types. For the particular case of a breather with up-down symmetry $\hat{S}_{u d}$, there are $k$ rotating vertical junctions in cells ( $i$ $+1), \ldots,(i+k)$ and two rotating horizontal junctions in the $(i)$ th and $(i+k)$ th cells, respectively. By making use of the flux quantization law (4), we obtain identical voltage drops across the vertical junctions in the resistive state. For the same reasons, the voltage drops across the horizontal junctions are two times smaller. Neglecting nonlinear contributions from the time average of $\sin \phi$ on resistive junctions we obtain

$$
\begin{gathered}
\alpha \frac{V}{2}=\frac{1}{\eta}\left\langle I_{i}^{m}\right\rangle, \\
\alpha V=\gamma+\left\langle I_{i+1}^{m}\right\rangle-\left\langle I_{i}^{m}\right\rangle, \\
\cdots \\
\alpha V=\gamma+\left\langle I_{i+k}^{m}\right\rangle-\left\langle I_{i+k-1}^{m}\right\rangle, \\
\alpha \frac{1}{2}=-\frac{1}{\eta}\left\langle I_{i+k}^{m}\right\rangle .
\end{gathered}
$$

Thus, the voltage drop across a resistive vertical junction that corresponds to the experimentally measured voltage drop across the ladder, is given by

$$
V=\frac{k \gamma}{\alpha(k+\eta)}
$$

Similarly, we analyze a breather with left-right symmetry $\hat{S}_{l r}$ and with inversion symmetry $\hat{S}_{i n}$. Taking into account that in these cases the voltage drops across resistive horizontal and vertical junctions are identical, we find

$$
V=\frac{k \gamma}{\alpha(k+2 \eta)} .
$$

In a similar manner, the result for a breather that has no symmetry [cf. Fig. 2(d)] reads

$$
V=\frac{k \gamma}{\alpha[k+(3 / 2) \eta]} .
$$

The above results for the dependence of the average voltage drop on the dc bias may be combined in a single expression

$$
V=\frac{k \gamma}{\alpha(k+[3-(1 / 2) \delta] \eta)},
$$

where $k$ is the number of vertical rotating junctions and $\delta$ denotes the number of resistive horizontal junctions. Note that $\delta=4$ for breathers with up-down symmetry, $\delta=2$ for left-right or inversion symmetry, and $\delta=3$ for no symmetry.

In order to analyze the interaction between the breather state and the linear EE's, we need to know the frequency $\Omega$ of a breather solution. Noting that the breather frequency is given by the lowest-realized voltage drop across a resistive junction, we find 
(1) Up-down symmetry

$$
\Omega=\frac{k \gamma}{2 \alpha(k+\eta)},
$$

(2) left-right symmetry and inversion symmetry

$$
\Omega=\frac{k \gamma}{\alpha(k+2 \eta)},
$$

(3) no symmetry

$$
\Omega=\frac{k \gamma}{\alpha(2 k+3 \eta)}
$$

\section{SPATIAL TAILS OF BREATHERS}

We consider the spatial dependence of Josephson phases in the presence of a breather state. At some distance from the breather center ("breather tail"), the Josephson phases librate with small amplitudes. In order to analyze the breather dynamics in the tail, we use the linearized system of equations (10). Keeping in mind the time periodicity of the breather solution, the librating Josephson phases take the form (for $n<0$, and with the breather center as the origin)

$$
\left(\begin{array}{c}
\varphi_{n}^{v} \\
\varphi_{n}^{h} \\
\tilde{\varphi}_{n}^{h}
\end{array}\right)=e^{\lambda n+i \Omega t}\left(\begin{array}{c}
\Delta_{v} \\
\Delta_{h} \\
\tilde{\Delta}_{h}
\end{array}\right) .
$$

Introducing the sum and difference variables of the amplitudes of Josephson phases of horizontal junctions

$$
\Delta_{h}^{+}=\frac{1}{2}\left(\Delta_{h}+\widetilde{\Delta}_{h}\right), \quad \Delta_{h}^{-}=\frac{1}{2}\left(\Delta_{h}-\widetilde{\Delta}_{h}\right)
$$

it follows that

$$
\begin{gathered}
\left(A-\frac{2}{\beta_{L}} \cosh \lambda\right) \Delta_{v}-\frac{2}{\beta_{L}}\left(1-e^{-\lambda}\right) \Delta_{h}^{-}=0, \\
-\frac{1}{\beta_{L} \eta}\left(1-e^{\lambda}\right) \Delta_{v}+B \Delta_{h}^{-}=0, \\
\left(-\Omega^{2}+i \alpha \Omega+1\right) \Delta_{h}^{+}=0
\end{gathered}
$$

where the frequency dependent parameters $A$ and $B$ are

$$
\begin{gathered}
A=-\Omega^{2}+i \alpha \Omega+\sqrt{1-\gamma^{2}}+\frac{2}{\beta_{L}}, \\
B=-\Omega^{2}+i \alpha \Omega+1+\frac{2}{\beta_{L} \eta} .
\end{gathered}
$$

From Eq. (25), we immediately find that $\Delta_{h}^{+}=0$, and hence, $\Delta_{h}^{-}=\Delta_{h}$. It follows that $\varphi_{n}^{h}=-\tilde{\varphi}_{n}^{h}$, i.e., breather tails appear with perfect up-down symmetry. This is at variance with the

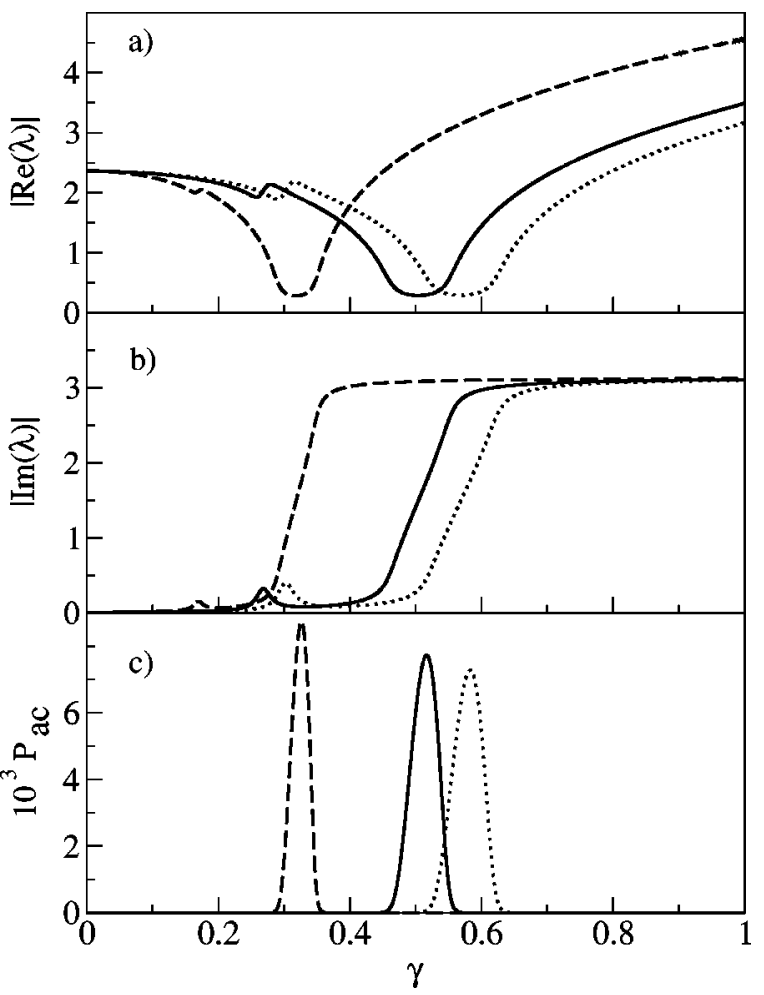

FIG. 3. Dependence of (a) real part $\operatorname{Re}(\lambda)$, (b) imaginary part $\operatorname{Im}(\lambda)$, and (c) $P_{a c}$ on $\gamma$ for different types of breathers: solid line-up-down symmetry, dashed line-left-right symmetry, dotted line -no symmetry. The parameters are $\alpha=0.1, \beta_{L}=3.0, \eta=0.35$, and $k=1$.

complex symmetry properties of the resistive breather center (see Sec. III). A nontrivial solution to the first two equations in Eq. (25) exists if

$$
A B-\frac{2}{\beta_{L}} B \cosh \lambda-\frac{4}{\beta_{L}^{2} \eta}+\frac{4}{\beta_{L}^{2} \eta} \cosh \lambda=0 .
$$

The dependence of the complex parameter $\lambda$ on the breather frequency $\Omega$ is given by

$$
\lambda=\ln \left(z+\sqrt{z^{2}-1}\right),
$$

with

$$
z=\frac{4-\beta_{L}^{2} \eta A B}{4-2 \beta_{L} \eta B} .
$$

Note here that this expression may be obtained directly from Eq. (13) by assuming that $q=i \lambda$ and substituting $\Omega^{2}-i \alpha \Omega$ instead of $\omega_{ \pm}^{2}$.

The real $\operatorname{Re}(\lambda)$ and imaginary $\operatorname{Im}(\lambda)$ parts of $\lambda$ determine, respectively, the spatial decay and spatial period of oscillations of Josephson phases in the breather tail. Moreover, $\operatorname{Re}(\lambda)$ and $\operatorname{Im}(\lambda)$ strongly depend on the breather frequency that in turn, may be changed by varying the external dc bias $\gamma$. In Figs. 3(a) and 3(b), we plot the real and imaginary parts of $\lambda$ for three breather types (cf. Fig. 2) versus $\gamma$. The minima of the real part of $\lambda$ correspond to resonances 


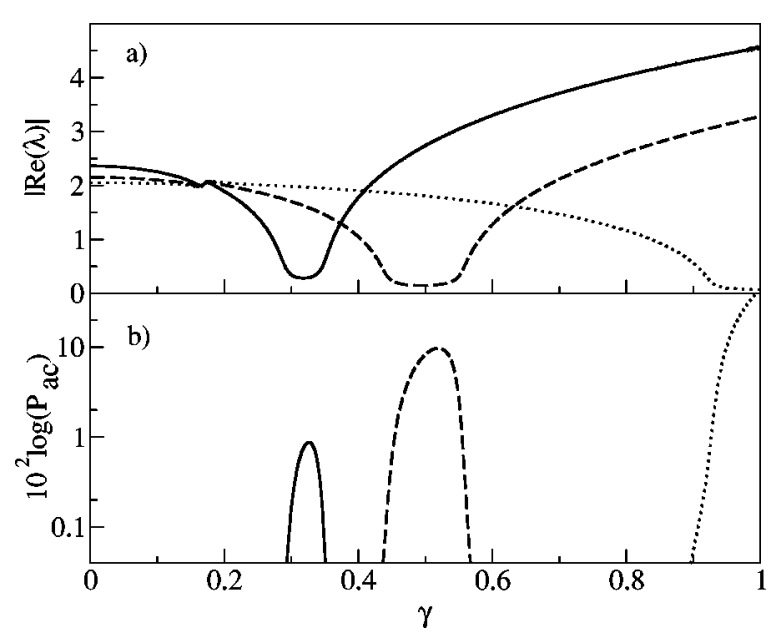

FIG. 4. (a) $\operatorname{Re}(\lambda)$ and (b) $P_{a c}$ versus $\gamma$ for different values of the inductance of the cell: $\beta_{L}=3$, solid line; $\beta_{L}=1$, dashed line; $\beta_{L}=0.2$, dotted line. All of these curves are for a left-right symmetry breather with $k=1$.

with linear EW's $\omega_{+}(q)$ and $\omega_{-}(q)$. As one can see, the current positions of these minima shift for different types of breathers. Note that the $\gamma$ range in the plots extends from zero to one. In fact, the breather will exist only in a narrower current region. This is due to the presence of both a finite nonzero retrapping current $[11,16]$ (switching to the superconducting state) and a particular current $\gamma<1$, where the breather switches to the HWS.

Since the EW frequencies $\omega_{+}(q)$ decrease with increasing inductance of the cell (13), the position of the global minimum also depends strongly on $\beta_{L}$ [see Fig. 4(a)].

Although the dynamics of the Josephson phases in the breather tail are completely determined by the dependence of the parameter $\lambda$ on the breather frequency, it may be more convenient to measure just the time-average power $P_{a c}$ of the libration of a junction at the edge of a JJL. We show below that the monitoring of $P_{a c}$ upon decreasing the dc bias allows one to develop a spectroscopy of EW's. The value of $P_{a c}$ is determined by the average kinetic energy of the edge vertical junction

$$
P_{a c}=\frac{1}{2}\left\langle\dot{\phi}_{l}^{v^{2}}\right\rangle, \quad l=-\frac{N}{2},
$$

and is derived in Appendix A. The typical dependences of $P_{a c}$ on the dc bias and the inductance of the cell for different types of breathers are presented in Figs. 3(c) and 4(b).

\section{BREATHER INSTABILITIES AND CLASSIFICATION OF RESONANCES}

In early experiments on breathers in JJL's [7,8], it was observed that breather states may switch to other breather states or HWS's upon variation of the dc bias $\gamma$. These switchings may be either due to the disappearance of the breather state as a solution of the underlying dynamical equations or due to the effect of dynamical instability of the breather state. In the latter case, the solution continues to exist, but turns unstable, forcing the system to search for another stable attractor state. The cause of such instabilities is the resonant interaction of the breather state with small amplitude (localized or delocalized) EE's.

In order to analyze the breather instabilities in more precise terms, we have to linearize the phase-space flow around a given breather solution and study the corresponding Floquet eigenvalue problem (see Appendix B). The outcome of this analysis is a spectrum of Floquet multipliers (i.e., eigenvalues) $\mu$. In most cases, the multipliers reside on a circle of radius

$$
R(\alpha)=\exp (-\alpha \pi / \Omega)
$$

in the complex plane, which is less than one. Thus, the multipliers may be expressed in the general form

$$
\mu=R(\alpha) \exp \left( \pm i \omega \frac{2 \pi}{\Omega}\right),
$$

where $\Omega$ is the breather frequency and $\omega$ is some characteristic EE frequency. The corresponding eigenvectors may be divided into two classes, namely, those that are localized on the breather and those that are delocalized. While it is more involved to make analytical predictions for the class of localized Floquet eigenstates, we may immediately proceed with the characterization of the class of delocalized eigenstates. Although the concrete form of the delocalized eigenvectors has to be obtained numerically, the fact that they are delocalized allows one to determine their frequency $\omega$. Indeed, since the breather is itself a localized state, delocalized Floquet eigenstates simply correspond to the abovediscussed linear EW's. Their frequencies have been derived in Sec. II, and thus, we may reconstruct the delocalized part of the Floquet spectrum using them.

Stable breathers are characterized by all Floquet multipliers being located inside or on the unit circle. Instabilities occur after collisions of multipliers on the inner circle with radius (31) and a subsequent detaching from this circle towards larger absolute values. Although in the dissipative case, an additional change of the control parameter (dc bias $\gamma$ ) is necessary in order for the corresponding Floquet eigenvalue(s) to cross and escape the unit circle, we may still look for collision conditions and classify possible instability scenarios.

Note that for Hamiltonian systems any collision and detaching from the unit circle leads to an instability. Finite (even though possibly weak) dissipation may drastically change the instability patterns by selecting the strong instabilities (large detachments) over the weak instabilities (small detachments). This is exactly what we observe in our numerical studies.

By applying the general stability analysis of nonlinear discrete systems [17] we obtain three possibilities for multiplier collisions. The first is realized when the collision takes place on the positive real axis in the complex plane. This implies that a multiplier is colliding with its complex conjugate partner. With Eq. (32), it follows that

$$
\omega=m \Omega,
$$


for any integer number $m$. These are primary resonances of the breather frequency or its higher harmonics with any of the frequencies of the EE's.

The collision of an eigenvalue with its complex conjugate partner may also take place on the negative real axis in the complex plane

$$
\omega=\left(\frac{1}{2}+m\right) \Omega .
$$

These are parametric resonances of the breather state with EE's.

The third case is realized when the collision takes place away from the real axis. Then a multiplier has to collide with a different one, but not with its own complex conjugate partner. It follows that

$$
\omega_{1} \pm \omega_{2}=m \Omega .
$$

This is a combination resonance, as the breather frequency (or its multiple) has to match a sum (difference) of the frequencies of two different $\mathrm{EE}$ frequencies.

We stress again that EE's may be localized or delocalized. While we may proceed with an analytical prediction of collisions using delocalized Floquet multipliers, we have to use numerical calculations to observe collisions involving localized ones. Especially the combination resonance may involve either two delocalized, two localized, or one delocalized and one localized Floquet eigenvalues. In addition, the above-mentioned dissipation-induced selection of weak and strong instabilities will result in some possible collisions being harmless (leaving the breather stable) while others will turn out to be important for understanding breather instabilities.

\section{NUMERICAL SIMULATIONS OF BREATHER DYNAMICS}

To study the breather dynamics, we performed direct numerical simulations of the set of equations (8). All simulations were carried out for JJL's with $N=10$ cells. We impose open boundary conditions and use the fourth-order RungeKutta method. Time is measured in dimensionless time units. The initial value of the dc bias was $\gamma=0.8$. We choose proper initial conditions that lead to the relaxation of the system into a particular breather state of left-right symmetry with one resistive vertical junction, as in Fig. 2(b). After a waiting time of 500 time units we use the next 500 time units to calculate the time-averaged characteristics of the state. We then decrease the dc bias $\gamma$ by a tiny step of $\Delta \gamma=0.0005$ and repeat the procedure. We checked that our results do not change upon further increase of the waiting time. We varied the anisotropy $\eta$ and the inductance of the cell $\beta_{L}$ while the dissipation $\alpha=0.1$ was fixed. We will comment on hysteresis effects due to additional increasing of the current in the conclusion.

There are three different ways to monitor the simulations. The first one is the $I-V$ characteristics, that is, the dependence of the averaged voltage drop across the resistive vertical junction on the dc bias. Furthermore, we obtain the

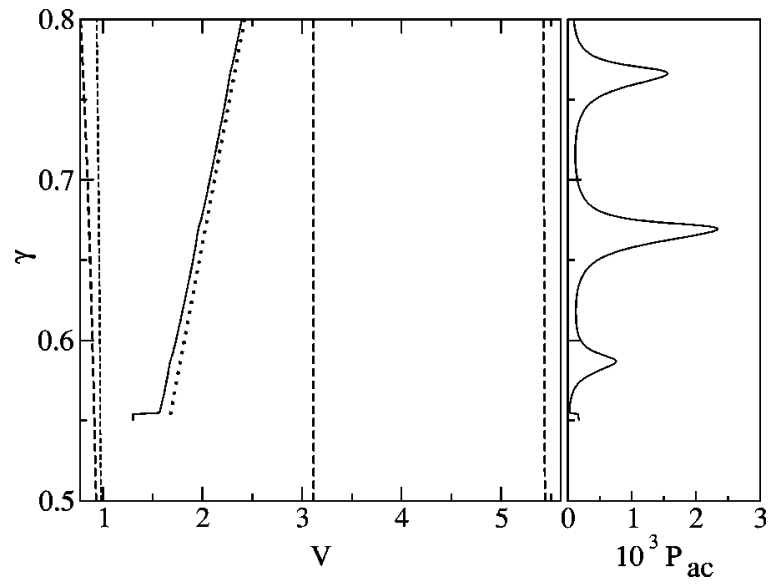

FIG. 5. $I-V$ characteristics and the $P_{a c}$ dependence on the dc bias current for $\alpha=0.1, \beta_{L}=0.2, \eta=1.15$.

power $P_{a c}(30)$ of ac oscillations of the vertical junction at the edge of JJL's. Finally, we generate time-resolved images (movies) of the full dynamical behavior of the ladder so that we may visually check whether the system still resides in the initially chosen breather state, or switches into another state. Our results are presented in Figs. 5-11, where each figure consists of two parts. In the left-hand parts, the $I-V$ characteristics are shown (solid lines) together with the approximate results from Sec. III (dotted lines). The vertically oriented dashed lines indicate the band edges of the linear EW's. In the right-hand part of the figures we show the dependence of $P_{a c}$ (solid lines) on the dc bias together with our approximate analysis from Sec. IV and Appendix A (dashed lines), where appropriate.

We start with the case of small $\beta_{L}$ values. For $\beta_{L}=0.2$ and $\eta=1.15$ (Fig. 5) the breather is easily excited, and its frequency is located below $\omega_{+}(q)$. In the $P_{a c}$ plot, we observe peaks that are due to the resonance of the second and third harmonics of the breather with $\omega_{+}(q)$. These resonances are primary ones, as discussed above. Note that their presence is barely seen on the $I-V$ curve. The series of observed peaks is related to the finite size of the system, and

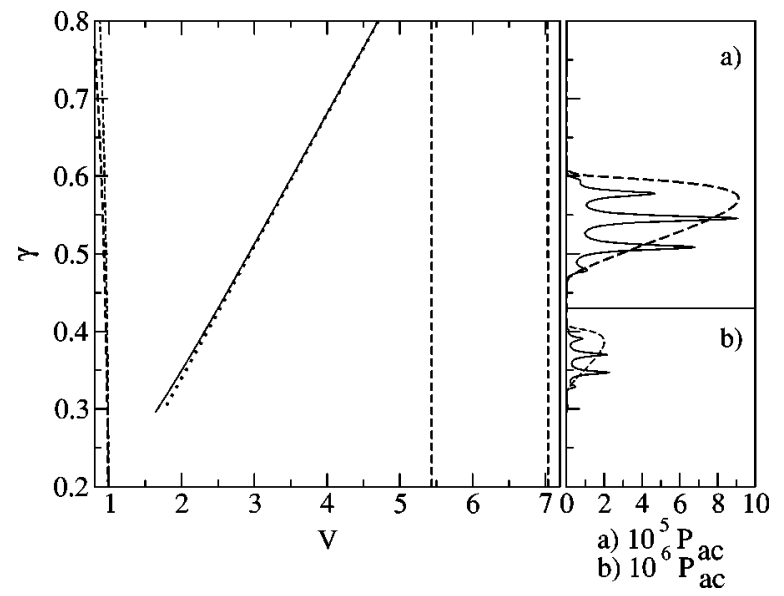

FIG. 6. $I-V$ characteristics and the $P_{a c}$ dependence on the dc bias current for $\alpha=0.1, \beta_{L}=0.2, \eta=0.35$. 


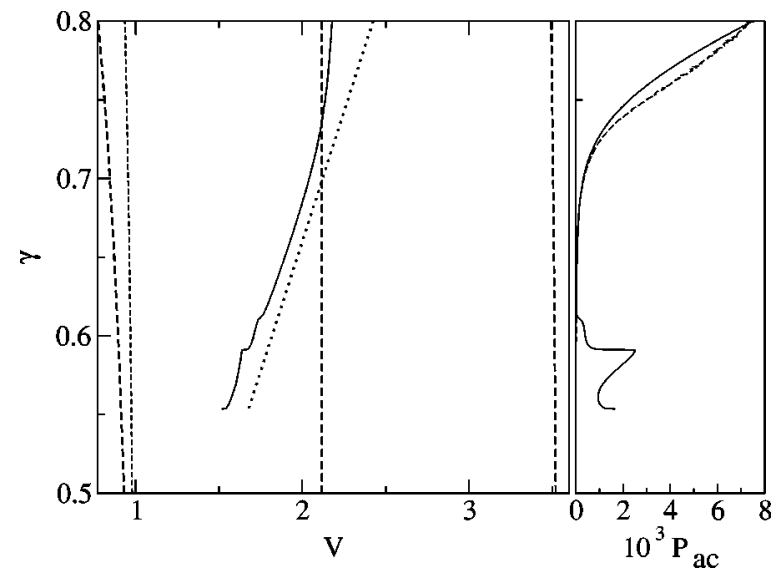

FIG. 7. $I-V$ characteristics and the $P_{a c}$ dependence on the dc bias current for $\alpha=0.1, \beta_{L}=0.5, \eta=1.15$.

therefore to the resonant interaction of the breather with a discrete set of cavity modes as was discussed at the end of Sec. II. We tested our interpretation by increasing the size of the system and observed the predicted increase in the number of resonance peaks. Close to the lowest possible current (around $\gamma=0.55$ ), we observe a switching to another breather state, which however has the same symmetry and spatial structure. Note that shortly after this switching (upon further lowering of the current) we lose the breather and the system switches to the superconducting ground state.

For lower values of the anisotropy $\eta=0.35$ (Fig. 6), the resonances are again not detectable in the $I-V$ curve. However, by monitoring $P_{a c}$, we observe the singularities that correspond to the primary resonance $2 \Omega=\omega_{+}(q)$. Moreover, at the dc bias $\gamma \approx 0.35$, we detect a weak third-order primary resonance $3 \Omega=\omega_{+}(q)$ in the breather tail. The dashed line in the right part of the figure is the prediction of $P_{a c}$ using our approximate tail analysis. Note that our approximate tail analysis is based on the assumption of a dense spectrum of EW's. Consequently, the calculated $P_{a c}$ presents an envelope of the numerically observed series of discrete peaks.

Next, we increased the inductance of the cell to $\beta_{L}=0.5$.

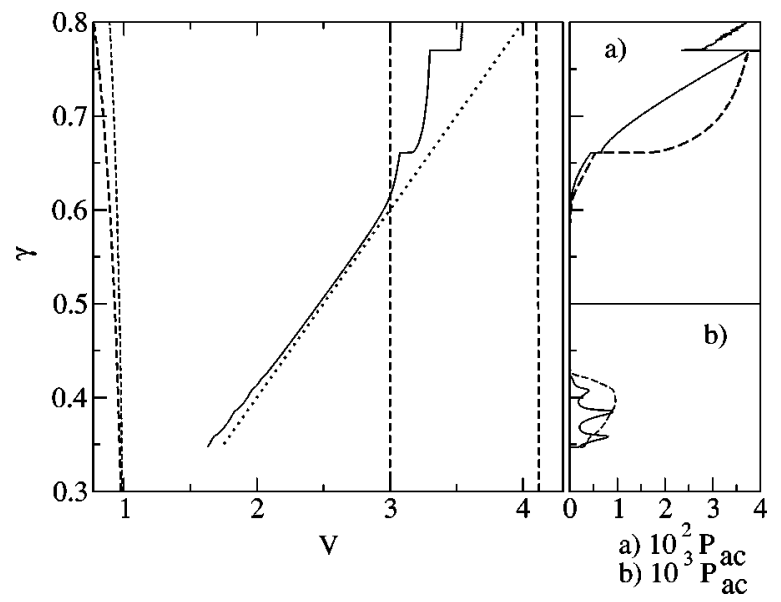

FIG. 8. $I-V$ characteristics and the $P_{a c}$ dependence on the dc bias current for $\alpha=0.1, \beta_{L}=0.5, \eta=0.5$.

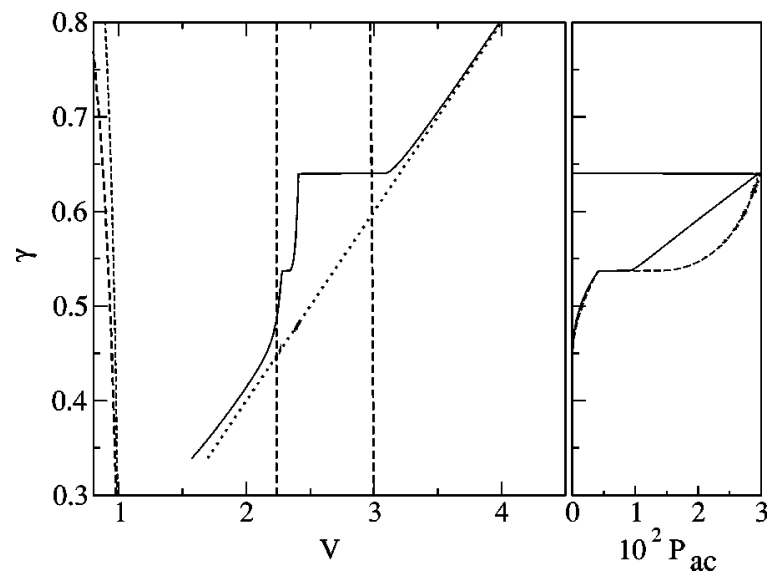

FIG. 9. $I-V$ characteristics and the $P_{a c}$ dependence on the dc bias current for $\alpha=0.1, \beta_{L}=1.0, \eta=0.5$.

In Fig. 7, we show the results for $\eta=1.15$. For the initial value of the dc bias $\gamma=0.8$, the breather frequency is already located inside the $\omega_{+}(q)$ band of EW's, and this primary resonance is observed in the $I-V$ curve. Indeed, the slope of the $I-V$ curve is larger than the prediction (21), which does not take into account resonant interactions with EE's. With decreasing dc bias, the breather frequency is lowered and the above primary resonance disappears. However, at lower current values the next primary resonances $2 \Omega=\omega_{+}(q)$ occur and are observable, both in the breather tail and in the $I-V$ characteristics.

The primary resonance structures $\left[\Omega=\omega_{+}(q)\right]$ in the large current domain are also observed for smaller values of the anisotropy parameter $\eta=0.5$ (Fig. 8). In this case, they manifest themselves through resonant steps in the $I-V$ curve [18]. At lower values of the dc bias, we again observe primary resonances with $m=2$.

For $\beta_{L}=1.0$ and $\eta=0.5$ (Fig. 9), the breather frequency is located above $\omega_{+}(q)$ for large current values. Upon decreasing the dc bias, we observe a peculiar switch to a different breather state with the same spatial structure but a lower frequency located inside $\omega_{+}(q)$. The most interesting feature here is that shortly before the switching, the breather fre-

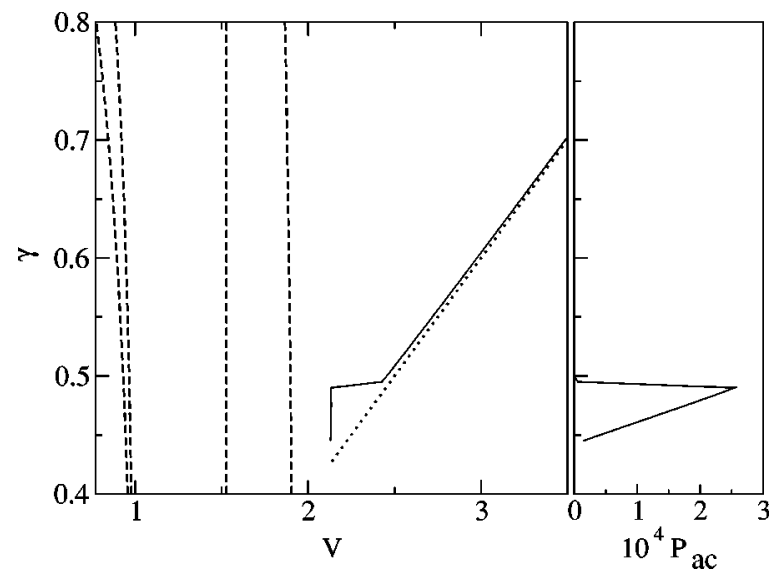

FIG. 10. $I-V$ characteristics and the $P_{a c}$ dependence on the dc bias current for $\alpha=0.1, \beta_{L}=3.0, \eta=0.5$. 


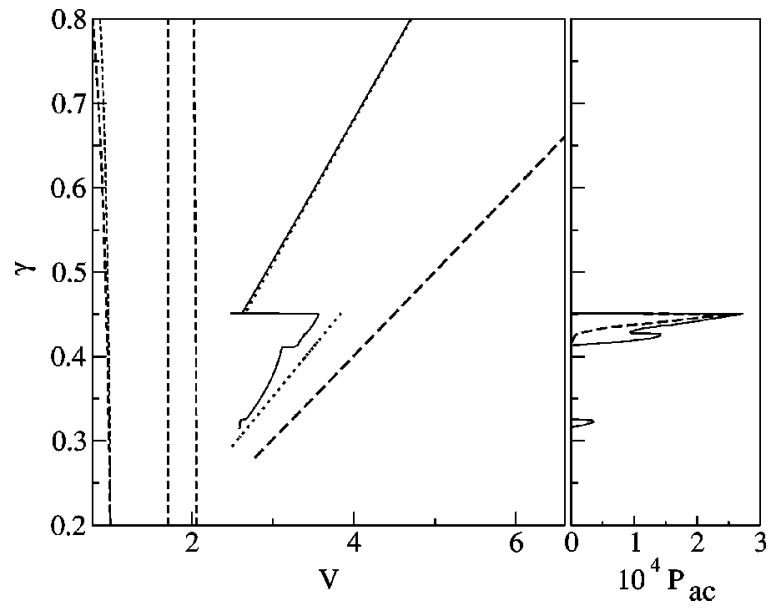

FIG. 11. $I-V$ characteristics and the $P_{a c}$ dependence on the dc bias current for $\alpha=0.1, \beta_{L}=3.0, \eta=0.35$.

quency is clearly larger and outside of the $\omega_{+}(q)$ region. Upon further lowering of the dc bias we observe primary resonances $\Omega=\omega_{+}(q)$, and corresponding resonant steps in the $I-V$ curve.

Let us increase the inductance of the cell $\beta_{L}$ even further. For $\beta_{L}=3$ and $\eta=0.5$ (Fig. 10) we again find that breather frequencies are located above $\omega_{+}$for large current values. Similar to the previous case, we observe a switching when the breather frequency is clearly outside (above) the branch $\omega_{+}(q)$. This brings the system into another breather state with the same spatial structure, but with a frequency again located above $\omega_{+}$. This highly nonlinear state is then lost by switching to the superconducting ground state after further decrease of the dc bias.

Now we come to an interesting observation. Lowering the anisotropy $\eta=0.35$ (Fig. 11), we again observe the switching at a breather frequency being located above $\omega_{+}(q)$. However, the switching increases the voltage drop. The state is of a different internal structure. We remind the reader that all previous numerical results have been obtained for a breather with a structure as in Fig. 2(b). Here we find that after the switch, the breather state is characterized by three vertical junctions being in the resistive state. At the same time the, symmetry is broken. In fact, this state exactly corresponds to the example given in Fig. 2(d). Note that similar switchings (which lead to an increase of the number of resistive junctions) have been reported in early experimental studies $[8,19]$. The left-right symmetry breaking leads to interesting features in the breather tails. The no-symmetry breather has a two times lower frequency than the voltage drop across the vertical junction. Thus, the breather frequency is inside the upper band $\omega_{+}(q)$, and a primary resonance is clearly observed in the $P_{a c}$ dependence on $\gamma$.

In order to test the influence of small fluctuations on the $I-V$ curves, we repeated the simulations in the presence of small noise with amplitude $\approx 10^{-8}$. All obtained results are stable except the switching outcome in Fig. 11. While this switching occurs at the same current value, the breather state is changed. In particular, we observed the left-right symmetry breather with three junctions being in the resistive state.
Thus, we find extreme sensitiveness of the outcoming breather structure (including its symmetries) to small fluctuations. This implies that the boundaries of the volumes of attraction of different (breather) attractors are entangled in a very peculiar way. We may reliably predict the switching position, but not the outcome of the switching.

We also numerically simulated the breather dynamics in the JJL with an extremely large inductance of the cell $\beta_{L}$ $=500$. We did not find any indication of resonances and instabilities. We argue that the reason for that is the weak dispersion of the linear EW's for such large values of $\beta_{L}$. This implies that interactions along the ladder are weak. The breather is continued to small current values until it switches to the superconducting state at the dc bias $\gamma=0.22$ for $\eta$ $=0.35$. This particular value may be obtained by making use of the simple dc analysis (17) and the standard theory of the retrapping current in a single small Josephson junction $[10,16]$

$$
\gamma_{r}=(1+2 \eta) \frac{4 \alpha}{\pi}
$$

This equation yields a value of 0.22 for the considered case, in good agreement with the numerical observation. Note that within this theory, retrapping occurs purely due to energy considerations, not due to resonances (or instabilities).

It is very important to notice that for cases with small or intermediate values of inductance of the cell (Figs. 5-11) the observed currents at which we lose the breather state and switch to the superconducting one exceed the expected retrapping values (36). We will explain this disagreement in the next section.

Motivated by the above findings, we investigated the loss of the HWS upon lowering the current. We recall that in this state, all vertical junctions are resistive and all horizontal ones are superconducting. Usually, it is assumed that the HWS loss is again due to a standard retrapping mechanism. It is important that any numerical simulation of such a process is done with the addition of some weak noise, because the processor will otherwise perform a perfect simulation of a single junction repeated $N+1$ times. We chose $\beta_{L}=3$ and $\eta=0.35$. The $I-V$ characteristic is shown by a thick dashed line in Fig. 11. The expected retrapping current $4 \alpha / \pi$ $=0.127$ is clearly not reached. Instead, we observe the loss of the HWS at $\gamma=0.273$. At the same time, it follows from Eqs. (8) that the HWS exists as a solution down to the retrapping current of a single junction, i.e., down to $\gamma$ $=0.127$ So in this case, we conclude that the numerically observed loss of the HWS at $\gamma=0.273$ is due to an instability. The HWS continues to exist as a solution down to the standard retrapping current, but it is an unstable state. This result is very important, since very often the current value of HWS loss in the absence of a magnetic field is used to estimate different parameters of the system assuming that the HWS is behaving similar to a single junction. Our results show that this is definitely not the case. 
TABLE I. Comparison of theoretical predictions of primary resonances with the numerical results from Fig. 6. First column: $k$. Second column: the spectrum of EW's $\omega_{+}\left(q_{k}\right)$ [cf. (14)]. Third column: $2 \Omega$ obtained from the peak positions of $P_{a c}(\gamma)$ in Fig. 6(a). Fourth column: $3 \Omega$ obtained from the peak positions of $P_{a c}(\gamma)$ in Fig. 6(b).

\begin{tabular}{cccc}
\hline \hline$k$ & $\omega_{+}\left(q_{k}\right)$ & $2 \Omega$ & $3 \Omega$ \\
\hline \hline 1 & 5.475 & & \\
2 & 5.581 & 5.594 & 5.590 \\
3 & 5.745 & & \\
4 & 5.949 & 5.947 & 5.945 \\
5 & 6.174 & & \\
6 & 6.400 & 6.387 & 6.384 \\
7 & 6.609 & & \\
8 & 6.788 & 6.769 & 6.772 \\
9 & 6.923 & & \\
10 & 7.008 & 6.960 & 6.958 \\
\hline \hline
\end{tabular}

\section{EVALUATION OF RESONANCES AND EWS SPECTROSCOPY}

This section is devoted to a quantitative explanation of the observed resonances and switchings.

\section{A. Primary resonances}

Primary resonances are characterized by $m \Omega=\omega$, where $\omega$ is some EE frequency. We detected various primary resonances with extended EW's. The case $m=1$, which corresponds to the breather frequency being located inside the $\omega_{+}(q)$ band, shows up with resonant steps in the $I-V$ curves (see, Figs. 8-9). The finite number of observed resonant steps is due to the discrete spectrum of the excited cavity modes. In addition, we observe strong variations of the breather tail amplitudes.

Higher-order primary resonances $(m=2,3)$ are much less pronounced in the $I-V$ characteristics. They mainly lead to a weaker localization of the breather tail and may be clearly detected in the form of sharp peaks in the $P_{a c}(\gamma)$ dependence. Since the breather in our case has left-right symmetry, the only linear cavity modes that may be exited are symmetric (see Sec. II). These modes are characterized by even values of $k$ in the expression $q_{k}$ (14). We start our evaluation of these resonances with the case shown in Fig. 6. We determine the $\gamma$ value of each observed peak in $P_{a c}(\gamma)$ and thus obtain the corresponding breather frequency $\Omega(\gamma)$. We then compare its multiples with the discrete spectrum of linear mode frequencies of the $\omega_{+}$branch. The numbers are listed in Table I. We find that all observed resonances are due to symmetric linear modes (even $k=2,4,6,8,10$ ) as expected.

The same method of analysis allows us to conclude that the three peaks in Fig. 5 (in decreasing order of dc bias) are due to the following resonances: $(m, k)=(2,6) ;(2,4) ;(3,8)$. Similarly, the shoulder and the peak in Fig. 6 are due to resonances with $(m, k)=(2,10) ;(2,8)$. Finally, the resonances in Fig. 8 correspond to the values $(m, k)=(2,8) ;(2,6) ;(2,4)$. Note that in all of these cases, the deviations between the theoretical and observed numbers are much less than the frequency difference between adjacent cavity modes.

\section{B. Parametric resonances}

So far, we did not comment on the nature of the switching from a breather state to the superconducting state for small and intermediate values of $\beta_{L}$. The Floquet analysis results show that all these switchings are due to an instability of the breather. In terms of Floquet multipliers, all of these instabilities are due to a collision of two localized Floquet multipliers on the negative real axis. The breather state continues to exist as a solution to the dynamical equations for lower current values, but it is unstable. Note that the so-called retrapping mechanism instead (as for a single junction) uses the critical current value as a criterion for retrapping. This argument is based purely on energy considerations and does not take into account any resonance mechanism. This is not surprising, as a single junction has no other degrees of freedom it may resonate with. Below the retrapping current, the resistive state disappears in this case. So, we may state that the switching from a breather state to the superconducting one as observed in our simulations is usually driven by resonances with localized EE's (frequency matching) and is not due to energy effects (current value matching).

\section{Combination resonances}

Let us discuss the nature of the switchings of the breather for intermediate $\beta_{L}$ values when the breather frequency is located above the branch $\omega_{+}(q)$. These switchings are again due to an instability. It is characterized by Floquet multipliers colliding away from the real axis. As discussed in Sec. V, this corresponds to a combination resonance. The numerical Floquet analysis shows that one of the two participating multipliers is a localized one [which bifurcates from the lower branch $\left.\omega_{-}(q)\right]$, while the second one belongs to the delocalized spectrum of $\omega_{+}(q)$. The Floquet multiplier that finally leaves the unit circle is a localized one. So again the instability of the breather is driven by a localized perturbation.

In Fig. 12, the dependence of the arguments and absolute values of all relevant Floquet multipliers is shown for the breather of Fig. 11. For convenience, we do not plot the complex conjugate multipliers and restrict the arguments to $0 \leqslant \arg (\mu) \leqslant \pi$. The narrow band $\omega_{-}(q)$ and broad band $\omega_{+}(q)$ are nicely observed. The degenerate band $\omega_{0}$ is located slightly above $\omega_{-}(q)$. This band does not interact with other multipliers when crossing them, as expected from our analytical considerations. The two separated arguments that are located below the $\omega_{-}(q)$ band have localized eigenvectors [21].

In the plot of the absolute values, we observe the predicted values $\mu=1$ and $e^{-\alpha T_{b}}$. The multipliers that correspond to lines between these two states generally reside on the circle with radius (31). Many of them depart from this circle due to collisions. At current values of $0.55<\gamma<0.7$, we observe parametric resonances $2 \Omega=\omega_{+}(q)$, which belong to the set of weak resonances and do not evolve into a global instability. However, it is possible that a slight variation of control parameters (e.g., decreasing the damping $\alpha$ ) 


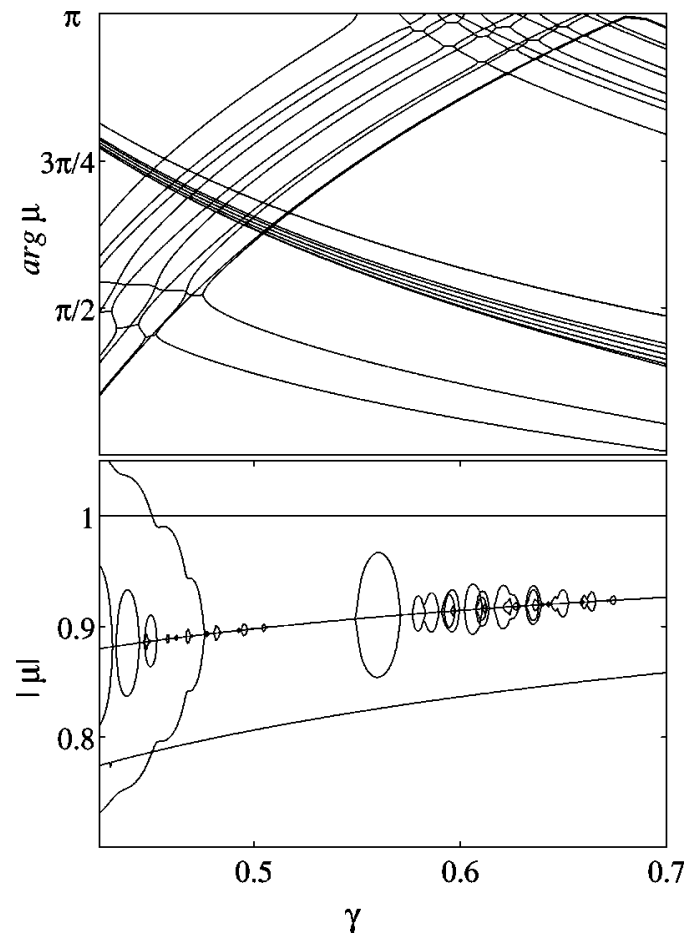

FIG. 12. Arguments and absolute values of Floquet multipliers versus $\gamma$ for the breather state in Fig. 11.

might change these resonances into strong ones. Then we would expect sudden instabilities of the breather state at these large current values. In our case, the global instability is realized when one of the localized multipliers collides with the $\omega_{+}(q)$ band around $\gamma=0.48$. Subsequent lowering of the current leads to a fast escape of this multiplier from the unit circle and to the observed switching.

The importance of localized EE's for the destabilization of a breather is simply due to the localized nature of the latter. It is hard (if not impossible) for a breather to generate a parametric instability through extended EW's alone, as these excitations are damped out far from the breather center. In contrast, localized EE's do not travel away from the breather center. These modes may be effectively excited by the breather, leading to an instability of the latter.

To understand the nature of the observed instability of the HWS, we show a similar Floquet multiplier plot in Fig. 13.

As in the previous plot, we observe weak parametric resonances of the upper EW band at current values $\gamma \sim 0.38$, which do not evolve into a global instability. Again, the observed instability is driven by a combination resonance at $\gamma=0.175$. Since the HWS is an extended state, all Floquet multipliers are also extended. The combination resonance is due to the collision of two Floquet multipliers belonging to the two EW branches $\omega_{ \pm}(q)$ with $q=\pi$ [we remind the reader that the EW spectrum of the HWS is different from that of the superconducting state and may be obtained by putting $\gamma=1$ in Eq. (13)]. Note that indeed for the present case, the frequency of the HWS at the instability equals 2.73 , while the value of the combination $\omega_{-}(\pi)+\omega_{+}(\pi)=2.69$. For current values $\gamma \sim 0.18$ the HWS is becoming stable again. However, around $\gamma=0.17$, another even stronger in-

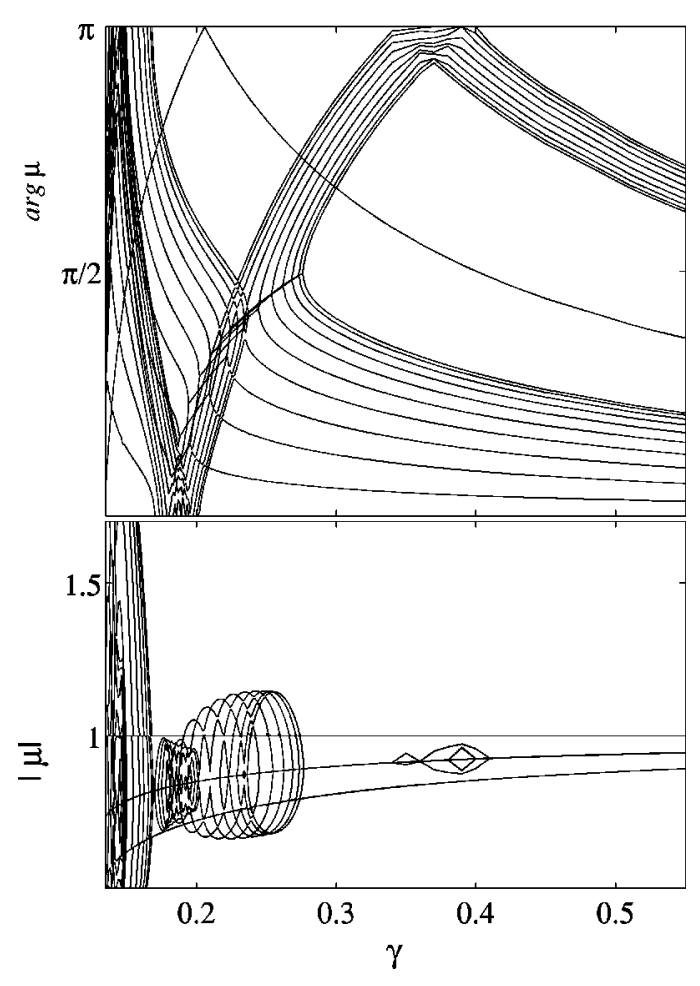

FIG. 13. Arguments and absolute values of Floquet multipliers versus $\gamma$ for the HWS in Fig. 11.

stability due to parametric resonance sets in, which brings the HWS to the next instability well above the expected retrapping current.

\section{CONCLUSION}

We have presented analytical and numerical studies of breather properties in Josephson junction ladders. Our results confirm and substantially extend early suggestions that breathers may resonate in different ways with localized and extended electromagnetic modes. The numerical studies have been done in a parallel manner to the way experiments are conducted. The variation of the control parameter $\gamma$ allows one to continuously change the breather frequency, whereas the linear mode spectrum is not significantly changed in the domain of interest. We observed primary resonances $m \Omega$ $=\omega_{+}\left(q_{k}\right)$ with extended EW's, parametric resonances $\Omega$ $=2 \omega$ with localized EE's, and combination resonances $m \Omega=\omega+\omega_{+}\left(q_{1}\right)$ with a localized EE and a delocalized EW participating. We also observed a combination resonance that leads to a switching from a small breather (one resistive vertical junction) to a larger one (three resistive vertical junctions) together with a possible symmetry lowering of the breather.

The primary resonances with extended EW's lead to singularities in the breather tails. This allows one to develop a spectroscopy of EW's by monitoring $P_{a c}$ versus $\gamma$. Such a spectroscopy may be experimentally realized, e.g., with the help of a well-known Josephson junction detector technique $[11,22,23]$. It could be important for obtaining a coherent source of high-frequency radiation, since in such a resonance, the whole breather tail starts to coherently oscillate 
with large amplitudes. The resistive breather center serves as a region of energy input via a dc bias.

Our studies show that the main control parameter (in addition to the dc bias) is the self-inductance $\beta_{L}$. For small $\beta_{L}$ values the breather frequency is located between the two branches of EW's, $\omega_{ \pm}(q)$. One may perform spectroscopy of EW's of the upper branch, or observe parametric instability of a breather due to localized modes. Moreover, as $\beta_{L}$ increases, resonant steps in the $I-V$ characteristics may be observed. For intermediate $\beta_{L}>1$ values, the breather frequency is located above $\omega_{+}(q)$. In this case, we observe combination resonances due to localized and delocalized EE's, which may result in an unusual sensitivity of the switching outcome on small fluctuations. Large $\beta_{L}$ values stabilize the breather states, make resonances impossible, and lead to a standard retrapping mechanism for breather switching to the superconducting ground state. This is likely the situation for the reported experimental data in Ref. [9]. However, lower $\beta_{L}$ values allow for the appearance of the above-listed resonances (and perhaps even other still unobserved resonances). We believe that our findings will help to make the proper parameter choice when designing other ladders for experiments.

Note that throughout our studies, we always decrease the dc bias $\gamma$. Let us consider a breather state No. 1, which becomes unstable upon lowering the dc bias at a certain value $\gamma_{1}$. Let this be a case where the system will switch to another stable breather state No. 2. This breather state is in fact keeping its stability not only upon further lowering of the dc bias, but also upon a reversing (increasing) of the dc bias. Thus, we find that there exist dc bias windows in which both the starting breather state No. 1 and the breather state No. 2 are stable. Even though their dc spatial structures (cf. Fig. 2) may be identical, the average voltage drops (and frequencies) are in general different. Further increasing of the dc bias while staying on the breather state No. 2 will lead to an instability and switching at $\gamma_{2}>\gamma_{1}$. In case the switching brings the system back to the breather state No. 1, we are faced with the well-known two-state hysteresis phenomenon in Josephson junction systems. However, we also observed cases when the switching due to an instability of breather state No. 2 (upon increasing the dc bias) brings the system to yet another state, which differs from breather state No. 1, e.g., simply to the HWS. In such a case, the hysteretic behavior is of a more complex nature. To keep the discussion of our studies as clear as possible we did not present data for increasing current.

Another observation is that the expected values of the retrapping current based on a pure dc analysis [10] are too low to match the observed values at which the breather switches to the superconducting ground state. Only for very large $\beta_{L}$ values do we observe agreement. For all other cases, the breather switches to the superconducting state via an instability driven by parametric resonance with localized EE's. Moreover, the HWS also undergoes an instability that is due to combination resonances with extended EW's.

In this paper we always started with a breather configuration as in Fig. 2(b), at large current values. It may be expected that the results for other starting configurations will show up with similar properties, and perhaps with additional types of resonances as well. This may be due to the fact that the structure of the phase space is very complex, being separated in many different regions of attraction of different attractors. It is this complexity that makes the understanding of breather properties both a fascinating and complicated undertaking.

\section{ACKNOWLEDGMENTS}

We thank A. Benabdallah, P. Binder, M. Schuster, and A. V. Ustinov for valuable discussions and F. V. Kusmartsev and J. J. Mazo for sending us their preprints prior to publication. This work was supported by the Deutsche Forschungsgemeinschaft FL200/8-1 and by the European Union under the RTN Project No. LOCNET HPRN-CT-199900163.

\section{APPENDIX A: POWER OF AC LIBRATIONS AT THE EDGE OF A JJL}

Here we derive the time-average power of ac librations at the edge of a JJL. This characteristic is proportional to the average kinetic energy $\left\langle\dot{\phi}^{2} / 2\right\rangle$. In order to obtain an expression for the kinetic energy, we have to determine the dynamics of the junction at the JJL edge. For this, we write the system of Eqs. (25) in a matrix form

$$
\hat{A} \vec{v}=0
$$

Here, $\vec{v}$ is an unknown vector

$$
\vec{v}=\left(\begin{array}{c}
\Delta_{v} \\
\Delta_{h}
\end{array}\right)
$$

and $\hat{A}$ is a $2 \times 2$ matrix

$$
\hat{A}(\lambda)=\left(\begin{array}{ll}
a_{11}(\lambda) & a_{12}(\lambda) \\
a_{21}(\lambda) & a_{22}(\lambda)
\end{array}\right),
$$

where $\quad a_{11}(\lambda)=A-\left(2 / \beta_{L}\right) \cosh \lambda, \quad a_{12}(\lambda)=-\left(2 / \beta_{L}\right)(1$ $\left.-e^{-\lambda}\right), \quad a_{21}(\lambda)=-\left(1 / \beta_{L} \eta\right)\left(1-e^{\lambda}\right)$, and $a_{22}(\lambda)=B$. A nonzero solution exists if the determinant of $\hat{A}$ vanishes: $\operatorname{det} \hat{A}\left(\lambda_{0}\right)=0$. The parameter $\lambda_{0}$ was determined in Sec. IV (28).

The components of the vector $\vec{v}$ satisfy the condition

$$
\Delta_{v}=-\frac{a_{22}}{a_{21}} \Delta_{h}
$$

To determine the components $V$ and $H$ separately, we have to impose an additional condition at the breather center. This condition is not known exactly due to the complex dynamics in the resistive breather center. Nevertheless, the ac librations in the breather tails are weakly depending on it. Here, we use the simplest normalization condition

$$
\left|\Delta_{v}\right|^{2}+\left|\Delta_{h}\right|^{2}=1
$$


Substituting Eq. (A4) into Eq. (A5), we obtain

$$
\begin{gathered}
\Delta_{h}=\frac{\left|a_{21}\right|^{2}}{\sqrt{\left|a_{21}\right|^{2}+\left|a_{22}\right|^{2}}}, \\
\Delta_{v}=-\frac{a_{22} \bar{a}_{21}}{\sqrt{\left|a_{21}\right|^{4}+\left|a_{22}\right|^{2}\left|a_{21}\right|^{2}}} .
\end{gathered}
$$

Due to the up-down symmetry in the breather tail, the dynamics of Josephson phases at the edge of the JJL may be written in the form

$$
\begin{aligned}
\varphi_{n}^{v}= & -\frac{\operatorname{Re}\left(a_{22} \bar{a}_{21}\right) e^{\operatorname{Re}\left(\lambda_{0}\right) n} \cos \left[\operatorname{Im}\left(\lambda_{0}\right) n+\Omega t\right]}{\sqrt{\left|a_{21}\right|^{4}+\left|a_{22}\right|^{2}\left|a_{21}\right|^{2}}} \\
& +\frac{\operatorname{Im}\left(a_{22} \bar{a}_{21}\right) e^{\operatorname{Re}\left(\lambda_{0}\right) n} \sin \left[\operatorname{Im}\left(\lambda_{0}\right) n+\Omega t\right]}{\sqrt{\left|a_{21}\right|^{4}+\left|a_{22}\right|^{2}\left|a_{21}\right|^{2}}}, \\
\varphi_{n}^{h} & =\frac{\left|a_{21}\right|^{2} e^{\operatorname{Re}\left(\lambda_{0}\right) n} \cos \left[\operatorname{Im}\left(\lambda_{0}\right) n+\Omega t\right]}{\sqrt{\left|a_{21}\right|^{2}+\left|a_{22}\right|^{2}}} .
\end{aligned}
$$

We finally obtain the expression for the average kinetic energy (for $n<0$ )

$$
\frac{1}{2}\left\langle\dot{\varphi}_{n}^{v^{2}}\right\rangle=\frac{\Omega^{2}\left|a_{22} \bar{a}_{21}\right|^{2} e^{\operatorname{Re}\left(\lambda_{0}\right) 2 n}}{4\left(\left|a_{21}\right|^{4}+\left|a_{22}\right|^{2}\left|a_{21}\right|^{2}\right)} .
$$

\section{APPENDIX B: LINEAR STABILITY OF THE BREATHER IN JJL}

The stability of periodic motion is analyzed with the help of the Floquet theory $[17,24]$. Linearizing the system (8) around a time-periodic breather solution, we obtain

$$
\begin{gathered}
\ddot{\epsilon}_{n}^{v}+\alpha \dot{\epsilon}_{n}^{v}+A_{n}^{v}(t) \boldsymbol{\epsilon}_{n}^{v}=\frac{1}{\beta_{L}}\left(\Delta \epsilon_{n}^{v}+\nabla \epsilon_{n-1}^{h}-\nabla \widetilde{\epsilon}_{n-1}^{h}\right), \\
\ddot{\epsilon}_{n}^{h}+\alpha \dot{\epsilon}_{n}^{h}+A_{n}^{h}(t) \epsilon_{n}^{h}=-\frac{1}{\eta \beta_{L}}\left(\nabla \epsilon_{n}^{v}+\epsilon_{n}^{h}-\tilde{\epsilon}_{n}^{h}\right), \\
\ddot{\tilde{\epsilon}}_{n}^{h}+\alpha \dot{\tilde{\epsilon}}_{n}^{h}+\widetilde{A}_{n}^{h}(t) \widetilde{\epsilon}_{n}^{h}=\frac{1}{\eta \beta_{L}}\left(\nabla \epsilon_{n}^{v}+\delta_{n}^{h}-\tilde{\epsilon}_{n}^{h}\right),
\end{gathered}
$$

where $A_{n}(t)$ are time-periodic coefficients determined by the given breather state.

The substitution

$$
\left(\begin{array}{c}
\boldsymbol{\epsilon}_{n}^{v} \\
\boldsymbol{\epsilon}_{n}^{h} \\
\tilde{\boldsymbol{\epsilon}}_{n}^{h}
\end{array}\right)=e^{-(1 / 2) \alpha t}\left(\begin{array}{c}
\boldsymbol{\kappa}_{n}^{v} \\
\boldsymbol{\kappa}_{n}^{h} \\
\tilde{\boldsymbol{\kappa}}_{n}^{h}
\end{array}\right)
$$

allows one to eliminate the dissipation

$$
\ddot{\kappa}_{n}^{v}+B_{n}^{v}(t) \kappa_{n}^{v}=\frac{1}{\beta_{L}}\left(\Delta \kappa_{n}^{v}+\nabla \kappa_{n-1}^{h}-\nabla \widetilde{\kappa}_{n-1}^{h}\right),
$$

$$
\begin{gathered}
\ddot{\kappa}_{n}^{h}+B_{n}^{h}(t) \kappa_{n}^{h}=-\frac{1}{\eta \beta_{L}}\left(\nabla \kappa_{n}^{v}+\kappa_{n}^{h}-\widetilde{\kappa}_{n}^{h}\right), \\
\ddot{\widetilde{\kappa}}_{n}^{h}+\widetilde{B}_{n}^{h}(t) \widetilde{\kappa}_{n}^{h}=\frac{1}{\eta \beta_{L}}\left(\nabla \kappa_{n}^{v}+\kappa_{n}^{h}-\widetilde{\kappa}_{n}^{h}\right),
\end{gathered}
$$

where $B_{n}(t)=-(1 / 4) \alpha^{2}+A_{n}(t)$. Introducing the variables

$$
z_{n}^{v}=\kappa_{n}^{v}, \quad z_{n}^{h}=\sqrt{\eta} \kappa_{n}^{h}, \quad \tilde{z}_{n}^{h}=\sqrt{\eta} \widetilde{\kappa}_{n}^{h},
$$

we find the system of equations

$$
\begin{gathered}
\ddot{z}_{n}^{v}+B_{n}^{v}(t) z_{n}^{v}=\frac{1}{\beta_{L}} \Delta z_{n}^{v}+\frac{1}{\beta_{L} \sqrt{\eta}}\left(\nabla z_{n-1}^{h}-\nabla \widetilde{z}_{n-1}^{h}\right), \\
\ddot{z}_{n}^{h}+B_{n}^{h}(t) z_{n}^{h}=-\frac{1}{\eta \beta_{L}}\left(z_{n}^{h}-\tilde{z}_{n}^{h}\right)-\frac{1}{\beta_{L} \sqrt{\eta}} \nabla z_{n}^{v}, \\
\ddot{\ddot{z}}_{n}^{h}+\widetilde{B}_{n}^{h}(t) \widetilde{z}_{n}^{h}=\frac{1}{\eta \beta_{L}}\left(z_{n}^{h}-\widetilde{z}_{n}^{h}\right)+\frac{1}{\beta_{L} \sqrt{\eta}} \nabla z_{n}^{v} .
\end{gathered}
$$

These equations describe a Hamiltonian system, namely,

$$
\begin{gathered}
\dot{\vec{z}}_{n}=\frac{\partial \mathcal{H}}{\partial \vec{p}_{n}}, \\
\dot{\vec{p}}_{n}=-\frac{\partial \mathcal{H}}{\partial \vec{z}_{n}}
\end{gathered}
$$

where $\vec{z}_{n}=\left(z_{n}^{v}, z_{n}^{h}, \widetilde{z}_{n}^{h}\right), \vec{p}_{n}=\left(p_{n}^{v}, p_{n}^{h}, \tilde{p}_{n}^{h}\right)$, and the Hamiltonian $\mathcal{H}\left(\vec{z}_{n}, \vec{p}_{n}, t\right)$ is

$$
\begin{aligned}
\mathcal{H}= & \frac{1}{2} \sum_{n}\left[p_{n}^{v^{2}}+p_{n}^{h^{2}}+\tilde{p}_{n}^{h^{2}}\right]+\frac{1}{2} \sum_{n}\left[B_{n}^{v} z_{n}^{v^{2}}+B_{n}^{h} z_{n}^{h^{2}}+\widetilde{B}_{n}^{h} \widetilde{z}_{n}^{h^{2}}\right] \\
& +\frac{1}{2 \beta_{L}} \sum_{n}\left(z_{n}^{v}-z_{n-1}^{v}\right)^{2}+\frac{1}{2 \beta_{L} \eta} \sum_{n}\left(z_{n}^{h}-\widetilde{z}_{n}^{h}\right)^{2} \\
& +\frac{1}{\beta \sqrt{\eta}} \sum_{n} z_{n}^{v}\left[z_{n-1}^{h}-\tilde{z}_{n-1}^{h}-z_{n}^{h}+\widetilde{z}_{n}^{h}\right] .
\end{aligned}
$$

Since the particular Hamiltonian may be represented in a general quadratic form, the symplectic product of two different trajectories $\left\{\vec{p}_{n}(t), \vec{z}_{n}(t)\right\}$ and $\left\{\vec{p}_{n}{ }^{\prime}(t), \vec{z}_{n}^{\prime}(t)\right\}$ does not change in time [17]

$$
\mathcal{I}=\sum_{n}\left[\vec{p}_{n}^{\prime}(t) \vec{z}_{n}(t)-\vec{p}_{n}(t) \vec{z}_{n}^{\prime}(t)\right]
$$

Rewriting our set of equations in the form

$$
\delta \dot{\overrightarrow{z_{n}}}=\frac{\partial^{2} \mathcal{H}}{\partial \vec{p}_{n}^{2}} \delta \vec{p}_{n}+\frac{\partial^{2} \mathcal{H}}{\partial \vec{p}_{n} \partial \vec{z}_{n}} \delta \vec{z}_{n}
$$




$$
-\delta \dot{\vec{p}}_{n}=\frac{\partial^{2} \mathcal{H}}{\partial \vec{z}_{n} \partial \vec{p}_{n}} \delta \vec{p}_{n}+\frac{\partial^{2} \mathcal{H}}{\partial \vec{z}_{n}^{2}} \delta \vec{z}_{n},
$$

and using the notation

$$
\mathcal{J}=\left(\begin{array}{cc}
0 & E \\
-E & 0
\end{array}\right)
$$

where $E$ is the identity matrix, we obtain

$$
\left(\begin{array}{c}
\delta \dot{\vec{p}}_{n} \\
\delta \dot{\vec{z}}_{n}
\end{array}\right)=\mathcal{J}^{-1} \nabla^{2} \mathcal{H}\left(\begin{array}{c}
\delta \vec{p}_{n} \\
\delta \vec{z}_{n}
\end{array}\right),
$$

where $\nabla^{2} \mathcal{H}$ is the Hessian of $\mathcal{H}$.

Let us consider the following map by integrating the Eqs. (B12) over one period $T_{b}$ of the initial solution:

$$
\left(\begin{array}{c}
\delta \vec{p}_{n}\left(T_{b}\right) \\
\delta \vec{z}_{n}\left(T_{b}\right)
\end{array}\right)=U\left(T_{b}\right)\left(\begin{array}{l}
\delta \vec{p}_{n}(0) \\
\delta \vec{z}_{n}(0)
\end{array}\right) .
$$

Since the form $\mathcal{I}$ is symplectic (B9), $U\left(T_{b}\right)$ is symplectic too. As a result, we find that the eigenvalues of $U\left(T_{b}\right)$ have to fulfill the condition that if $\nu$ is an eigenvalue then $1 / \nu, \nu^{*}$, and $1 / \nu^{*}$ are also eigenvalues. Note that for a marginally stable periodic motion of a Hamiltonian system, the Floquet eigenvalues $\nu$ are located on the unit circle. Switching to an unstable state is realized by collisions of eigenvalues on the unit circle and departing from it.

All of the obtained relations for eigenvalues (and eigenvectors) may be rewritten for the original $\delta_{n}(t)$ variables (call the corresponding Floquet eigenvalues $\mu$ ). First of all, we expect that most of the eigenvalues will be located on a circle of radius (31). Further, it follows that if $\mu$ is an eigenvalue then $e^{-\alpha T_{b} / \mu, \mu^{*}}$, and $e^{-\alpha T_{b} / \mu^{*}}$ are eigenvalues too. So, all scenarios of collisions of the eigenvalues are similar to the Hamiltonian case. For a periodic motion to be stable, we need all eigenvalues $\mu$ to have absolute values less or equal to one, i.e., the complex numbers $\mu$ should reside inside the unit circle in the complex plane. Since the radius $R(\alpha) \leqslant 1$, it needs further finite variation of the control parameters (after a collision) to enforce an eigenvalue to traverse the finite distance to the unit circle and to exit it. Thus, not every collision will lead to an instability. However, the decrease of the dissipation parameter $\alpha$ may tune the system closer to the Hamiltonian case, making its states more sensitive to any occurring collisions. Note that due to the periodicity of the breather state, there is always one eigenvalue $\mu=1$, whose eigenvector is tangent to the breather orbit. Consequently, we always find another eigenvalue $\mu$ $=e^{-\alpha T_{b}}$ that is located on the positive real axis inside the unit circle. During all numerical computations of Floquet eigenvalues and eigenvectors, the above properties were tested and complete agreement was found.
[1] S. Strogatz, Nonlinear Dynamics and Chaos: with Applications to Physics, Biology, Chemistry and Engineering (Addison-Wesley, Reading, MA, 1994).

[2] A. J. Sievers and J. B. Page, Dynamical Properties of Solids VII Phonon Physics (Elsevier, Amsterdam, 1995).

[3] S. Aubry, Physica D 103, 201 (1997).

[4] S. Flach and C. R. Willis, Phys. Rep. 295, 181 (1998).

[5] B. Swanson, J. Brozik, S. Love, G. Strouse, A. Shreve, A. Bishop, W. Z. Wang, and M. Salkola, Phys. Rev. Lett. 82, 3288 (1999).

[6] U. Schwarz, L. Q. English, and A. J. Sievers, Phys. Rev. Lett. 83, 223 (1999).

[7] E. Trias, J. J. Mazo, and T. P. Orlando, Phys. Rev. Lett. 84, 741 (2000).

[8] P. Binder, D. Abraimov, A. V. Ustinov, S. Flach, and Y. Zolotaryuk, Phys. Rev. Lett. 84, 745 (2000).

[9] P. Binder, D. Abraimov, and A. V. Ustinov, Phys. Rev. E 62, 2858 (2000).

[10] E. Trias, J. J. Mazo, A. Brinkman, and T. P. Orlando, Physica D 156, 98 (2001).

[11] K. K. Likharev, Dynamics of Josephson Junctions and Circuits (Gordon and Breach, New York, 1986).

[12] P. Caputo, M. V. Fistul, A. V. Ustinov, B. A. Malomed, and S. Flach, Phys. Rev. B 59, 14050 (1999).

[13] S. Flach and M. Spicci, J. Phys.: Condens. Matter 11, 321 (1999).

[14] J. R. Phillips, H. S. J. van der Zant, J. White, and T. P. Ornaldo, Phys. Rev. B 47, 5219 (1993).
[15] G. Grimaldi, G. Filatrella, S. Pace, and U. Gambardella, Phys. Lett. A 223, 463 (1996).

[16] A. Barone and G. Paterno, Physics and Applications of the Josephson Effect (Wiley, New York, 1982).

[17] V. I. Arnold, Mathematical Methods of Classical Mechanics (Springer, Berlin, 1989).

[18] Recently, similar resonant steps in $I-V$ curves were observed experimentally by M. Schuster, P. Binder, and A. V. Ustinov (to be published).

[19] Similar switchings with an increasing number of resistive junctions have been obtained in Ref. [20] for a rather different set of dynamic equations. Such switchings have not been found in extensive numerical simulations in Ref. [10]. We think that this difference is due to the different step size of the dc bias used. We found that one has to use sufficiently small step sizes in order to observe these switchings.

[20] R. T. Giles and F. V. Kusmartsev, Phys. Lett. A 287, 289 (2001).

[21] Note that the frequencies of these two localized EE's can be nicely predicted taking into account the dynamics of two cells around the resistive breather center. This will be published elsewhere.

[22] S. P. Benz and C. J. Burroughs, Appl. Phys. Lett. 58, 2162 (1991).

[23] V. P. Koshelets, S. V. Shitov, A. V. Shchukin, L. V. Filippenko, J. Mygind, and A. V. Ustinov, Phys. Rev. B 56, 5572 (1997).

[24] S. Watanabe, H. S. J. van der Zant, S. H. Strogatz, and T. P. Orlando, Physica D 97, 429 (1996). 